\title{
Synthesis of Coumarin Derivatives using Glutamic Acid under Solvent-free Conditions
}

\section{SETAREH SABETPOOR and FARHAD HATAMJAFARI*}

\author{
Department of Chemistry, Faculty of Science, \\ Islamic Azad University-Tonekabon Branch, Tonekabon, Iran. \\ ${ }^{*}$ Corresponding author E-mail: hatamjafari@yahoo.com \\ http://dx.doi.org/10.13005/ojc/300265
}

(Received: March 05, 2014; Accepted: March 31, 2014)

\begin{abstract}
An efficient,simple and one-pot protocol for synthesis of coumarin derivatives on the basis condensation reaction of phenols with ethyl acetoacetate employing glutamic acid as a novel catalyst is described.
\end{abstract}

Key words: One-pot,Glutamic acid, Coumarins, Solvent-Free Conditions.

\section{INTRODUCTION}

Multicomponent reactions are efficient methods in thesynthesis of heterocycles. In Multicomponent reactions synthesis to produce massive amounts of waste, according to the isolation of the complex, toxic and hazardous solvent at each step andwill discovered, economically and environmentallyfriendly. Multicomponent reactions advantages, synthesized of heavy compound molecules by the reaction of small molecule ${ }^{1}$.

Coumarins derivatives are heterocyclic units in the field of natural and synthetic organic chemistry due to their wide range of biological and therapeutic properties such asanti-inflammatory, anti-tumor, antioxidant,anti-viral and anti-bacterial activities.Recently, coumarin appropriate analogue functionalized gland known as antibiotics agents, and receptor antagonists have emerged. In addition, several flavonoid containing the coumarin core unit have been isolated from natural sources also show interesting biological properties. However, Thesereactions often require harsh conditions and was happened long reaction time and low efficiency. Kinds of phenols used to replace for synthesiscoumarins ${ }^{2-9}$. Although numerous methods are capable of affecting these synthesis has been previously reported. Glutamic acidhas been used previously as a catalyst for synthesis of organic compound ${ }^{10}$, Previously, we have synthesized a number of heterocyclic compounds ${ }^{11}$. ${ }^{23}$. Herein we report glutamic acida new catalyst for the synthesis of Coumarins at one pot reaction, environmentally friendly easy separation with high yields(Scheme 1). 


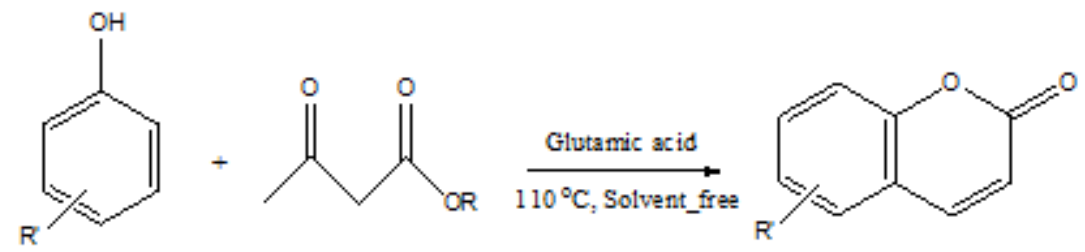

Scheme 1:

All chemicals were obtained from Merck or Flukawithout further purification. Silica gel SILG/ UV 254 plates were used for TLC. IR spectra were measured on a Shimadzu IR-470 Spectrophotometer. ${ }^{1} \mathrm{H}$ NMR spectra were determined on Bruker400 DRX AVANCE instrument at $400 \mathrm{MHz}$, respectively.

Typical procedure adopted for the synthesis of 7hydroxy-4-methyl-2H-chromen-2-one (S1)

A mixture of resorsinol $(1 \mathrm{mmol})$, ethyl acetoacetate $(1 \mathrm{mmol})$, and glutamic acid $(20 \mathrm{~mol} \%)$ was stirred at $110^{\circ} \mathrm{C}$ for a $15 \mathrm{~min}$. The progress of the reaction was monitored by using TLC. After completion of the reaction, the solid catalyst (glutamic acid) was washed with water, and finally purified by recrystallization in ethanol/water.

Data of compounds (S1-S5)

\section{7-hydroxy-4-methyl-2H-chromen-2-one (S1)}

White powder, Yield: (93\%), mp: $187-189^{\circ} \mathrm{C}$

IR $\left(v_{\text {max }} / \mathrm{cm}^{-1}\right)(\mathrm{KBr}): \quad 3440(\mathrm{OH}), \quad 1683(\mathrm{C}=\mathrm{O})$, $1604(\mathrm{C}=\mathrm{C}) \mathrm{cm}^{-1}$

${ }^{1} \mathrm{H} \mathrm{NMR}\left(400 \mathrm{MHz}\right.$; DMSO- $\left.d_{6}\right) \delta=2.34(3 \mathrm{H}$, $\left.\mathrm{s}, \mathrm{CH}_{3}\right), 6.10(1 \mathrm{H}, \mathrm{s}, \mathrm{CH}), 6.68\left(1 \mathrm{H}, \mathrm{d}, \mathrm{J}=2.0 \mathrm{~Hz}, \mathrm{H}_{\mathrm{c}}\right)$, $6.79\left(1 \mathrm{H}, \mathrm{d}, \mathrm{J}=2.0 \mathrm{~Hz}, \mathrm{H}_{\mathrm{b}}\right), 7.76(1 \mathrm{H}, \mathrm{d}, \mathrm{J}=8.8 \mathrm{~Hz}$, $\left.\mathrm{H}_{\mathrm{a}}\right), 8.85(1 \mathrm{H}, \mathrm{brs}, \mathrm{OH})$.

dihydroxy-4-methyl-2H-chromen-2-one (S2)7,8 White powder, Yield: (90\%), mp: $241-243^{\circ} \mathrm{C}$ IR $\left(v_{\text {max }} / \mathrm{cm}^{-1}\right)(\mathrm{KBr}): \quad 3444(\mathrm{OH}), \quad 1677(\mathrm{C}=\mathrm{O})$, $1600(\mathrm{C}=\mathrm{C}) \mathrm{cm}^{-1}$

${ }^{1} \mathrm{H} \operatorname{NMR}(\mathrm{DMSO}) \mathrm{v}=2.34\left(3 \mathrm{H}, \mathrm{s}, \mathrm{CH}_{3}\right)$, 6.11(1H, s, CH), 6.83-7.09(1H, m, $\left.\mathrm{H}_{\text {arom }}\right), 6.82(1 \mathrm{H}$, $\left.\mathrm{d}, \mathrm{J}=8.0 \mathrm{~Hz}, \mathrm{H}_{\mathrm{a}}\right), 7.09\left(1 \mathrm{H}, \mathrm{d}, \mathrm{J}=8.0 \mathrm{~Hz}, \mathrm{H}_{\mathrm{b}}\right), 9.30(1 \mathrm{H}$, brs, $\mathrm{OH}), 10.0(1 \mathrm{H}, \mathrm{brs}, \mathrm{OH})$.
5,7-dihydroxy-4-methyl-2H-chromen-2-one (S3)

White powder, yield (87\%), mp: $281-282^{\circ} \mathrm{C}$ IR $\left(v_{\text {max }} / \mathrm{cm}^{-1}\right)(\mathrm{KBr}): 3498,3440(2 \mathrm{OH}), 1674(\mathrm{C}=\mathrm{O})$, 1602 $(\mathrm{C}=\mathrm{C})$

${ }^{1} \mathrm{H} \operatorname{NMR}(\mathrm{DMSO}) \quad \mathrm{v}=2.47\left(3 \mathrm{H}, \mathrm{s}, \mathrm{CH}_{3}\right)$, 5.82(1H, s, CH), 6.16(1H, d, J=2.4 Hz, $\left.\mathrm{H}_{\mathrm{a}}\right), 6.26(1 \mathrm{H}$, $\left.\mathrm{d}, \mathrm{J}=2.4 \mathrm{~Hz}, \mathrm{H}_{\mathrm{b}}\right), 10.37(2 \mathrm{H}$, brs, $2 \mathrm{OH})$.

7-hydroxy-4,8-dimethyl-2H-chromen-2-one (S4) White powder, yield (89\%), mp: $261-263^{\circ} \mathrm{C}$ IR $\left(v_{\max } / \mathrm{cm}^{-1}\right)(\mathrm{KBr}): 3494(\mathrm{OH}), 1670(\mathrm{C}=\mathrm{O}), 1606$ $(\mathrm{C}=\mathrm{C})$

${ }^{1} \mathrm{HNMR}(\mathrm{DMSO}): \ddot{a}=2.27,2.54(6 \mathrm{H}, \mathrm{s}$, $\left.2 \mathrm{CH}_{3}\right), 6.03(1 \mathrm{H}, \mathrm{s}, \mathrm{CH}), 6.56\left(1 \mathrm{H}, \mathrm{d}, \mathrm{J}=0.8 \mathrm{~Hz}, \mathrm{H}_{\mathrm{a}}\right)$, $6.60\left(1 \mathrm{H}, \mathrm{d}, \mathrm{J}=0.8 \mathrm{~Hz}, \mathrm{H}_{\mathrm{b}}\right)$.

\section{4-methyl-2H-chromen-2-one (S5)}

White powder, Yield: (83\%), mp: $80-82^{\circ} \mathrm{C}$ IR $\left(v_{\max } / \mathrm{cm}^{-1}\right)(\mathrm{KBr}): \quad 1683(\mathrm{C}=\mathrm{O}), 1604(\mathrm{C}=\mathrm{C}) \mathrm{cm}^{-1}$

${ }^{1} \mathrm{H} \mathrm{NMR}\left(400 \mathrm{MHz}\right.$; DMSO- $\left.d_{6}\right) \delta=2.34(3 \mathrm{H}$, $\left.\mathrm{s}, \mathrm{CH}_{3}\right), 6.10(1 \mathrm{H}, \mathrm{s}, \mathrm{CH}), 6.68\left(1 \mathrm{H}, \mathrm{d}, \mathrm{J}=2.0 \mathrm{~Hz}, \mathrm{H}_{\mathrm{c}}\right)$, $6.79\left(1 \mathrm{H}, \mathrm{d}, \mathrm{J}=2.0 \mathrm{~Hz}, \mathrm{H}_{\mathrm{b}}\right), 7.76\left(1 \mathrm{H}, \mathrm{d}, \mathrm{J}=8.8 \mathrm{~Hz}, \mathrm{H}_{\mathrm{a}}\right)$.

\section{RESULTS AND DISCUSSION}

Herein, we report glutamic acid ascatalyst which could provide high yield, an efficient, environmentally friendly, easy separationand simple route for thesynthesis of coumarins.

\section{ACKNOWLEDGEMENTS}

We gratefully acknowledge the financialsupport from the Research Council of TonekabonBranch Islamic Azad University. 


\section{REFERENCES}

1. Balme G., Bossharth $\mathrm{E}$ andMonteiro N.,Eur. J. Org.Chem., 2003. ,21: 4101

2. Kostoval., Cur. Med. Chem., 2005.,5: 29

3. Al-AmieryA. A., KadhumA andMohamadA., Molecules, 2012.,17: 5713

4. ShikishimaY., TakaishiY., Honda G andet al., Chem. Pharm. Bull., 2001.,49: 877

5. Manolovl., Maichle-Moessmer $\mathrm{C}$ and Danchev N., Eur. J. Med. Chem., 2006. 41: 882

6. KontogiorgisC. A andHadjipavlou-LitinaD. J.,J. Med. Chem., 2005. 48: 6400

7. FylaktakidouK. C., Hadjipavlou-LitinaD. J., LitinasK. E andNicolaidesD. N., Cur. Pharm. Design., 2004. 10: 3813

8. BabaM., JinY., Mizuno A and et al., Bio. Pharm. Bull. 2002., 25: 244

9. Thornes D., DalyL., Lynch G and et al., Eur. J. Surgl. Oncol. 1989.,15: 431

10. Hatamjafari F and Abbasi E., Orient. J. Chem. 2013., 29:731

11. Azizian J., Hatamjafari F., Karimi A. R. and Shaabanzadeh M., Synthesis, 2006., 5: 765

12. Azizian J., Shaabanzadeh M., Hatamjafari F. and Mohammadizadeh M.R., Arkivoc, (xi): 2006. 47

13. Hatamjafari F., Synthetic Communications, 2006., ý36: 3563

14. Azizian J., Hatamjafari F. and Karimi A. R., Journal of Heterocyclic Chemistry, 2006.,43:1349

15. Hatamjafari F and Montazeri N., Turkish Journal of Chemistryý,2009.,33: 797

16. Bidram A., Hatamjafari $F$ and Doryeh A., Orient. J. Chem. 2013., 29: 123

17. Hatamjafari F., Orient. J. Chem., 2012. 28: 141

18. Hatamjafari F., Orient. J. Chem., 2013.29: 93

19. Hatamjafari F and Alijanichakoli F., Orient. J.Chem. 2013.,29: 145

20. Hatamjafari F and Hosseinian A., Orient. J.Chem., 2013.29: 109

21. Hatamjafari $F$ and Keyhani A., Orient. J.Chem. 2013.,29: 783

22. Hatamjafari F and Khojastehkouhi H., Orient. J. Chem. 2014., 30(1), 329-331.

23. Hatamjafari F and GermaniNezhad F., Orient. J. Chem., 2014. 30(1), 355-357. 